\title{
The Effects of Vitamin D on Gentamicin-Induced Acute Kidney Injury in Experimental Rat Model
}

\author{
Ender Hur, ${ }^{1}$ Alev Garip, ${ }^{2}$ Asuman Camyar, ${ }^{2}$ Sibel Ilgun, ${ }^{2}$ Melih Ozisik, ${ }^{2}$ Sena Tuna, \\ Murat Olukman, ${ }^{3}$ Zehra Narli Ozdemir, ${ }^{2}$ Eser Yildirim Sozmen, ${ }^{4}$ Sait Sen, ${ }^{5}$ \\ Fehmi Akcicek, ${ }^{2}$ and Soner Duman ${ }^{2}$ \\ ${ }^{1}$ Department of Nephrology, Bulent Ecevit University Medical School, Esenkoy, 67100 Zonguldak, Turkey \\ ${ }^{2}$ Department of Internal Medicine, Ege University Medical School, 35100 Izmir, Turkey \\ ${ }^{3}$ Department of Pharmacology, Ege University Medical School, 35100 Izmir, Turkey \\ ${ }^{4}$ Department of Biochemistry, Ege University Medical School, 35100 Izmir, Turkey \\ ${ }^{5}$ Department of Pathology, Ege University Medical School, 35100 Izmir, Turkey
}

Correspondence should be addressed to Ender Hur; hurender@hotmail.com

Received 5 April 2013; Revised 28 May 2013; Accepted 30 May 2013

Academic Editor: Hulya Taskapan

Copyright (C) 2013 Ender Hur et al. This is an open access article distributed under the Creative Commons Attribution License, which permits unrestricted use, distribution, and reproduction in any medium, provided the original work is properly cited.

\begin{abstract}
Introduction. Acute kidney injury (AKI) pathogenesis is complex. Findings of gentamicin nephrotoxicity are seen in $30 \%$ of the AKI patients. Vitamin D has proven to be effective on renin expression, inflammatory response, oxidative stress, apoptosis, and atherosclerosis. We aimed to investigate the effect of vitamin D in an experimental rat model of gentamicin-induced AKI. Methods. Thirty nonuremic Wistar albino rats were divided into 3 groups: Control group, $1 \mathrm{~mL}$ saline intramuscular (im) daily; Genta group, gentamicin $100 \mathrm{mg} / \mathrm{kg} /$ day (im); Genta + vitamin D, gentamicin $100 \mathrm{mg} / \mathrm{kg} /$ day (im) in addition to $1 \alpha, 25(\mathrm{OH})_{2} \mathrm{D}_{3}$ $0.4 \mathrm{mcg} / \mathrm{kg} /$ day subcutaneously for 8 days. Blood pressures and 24-hour urine were measured. Blood urea and creatinine levels and urine tubular injury markers were measured. Renal histology was semiquantitatively assessed. Results. Urea, creatinine and urine neutrophil gelatinase-associated lipocalin, and kidney injury molecule-1 were all increased in Genta group indicating AKI model. Systolic blood pressure decreased, but urine volume and glutathione increased in Genta + Vit D group compared to Control group. Histological scores indicating tubular injury increased in Genta and Genta + Vit D groups. Conclusions. Vitamin D does not seem to be effective on histological findings although it has some beneficial effects via RAS system and a promising effect on antioxidant system.
\end{abstract}

\section{Introduction}

Acute kidney injury (AKI) pathogenesis is complex, and promoting events may be completely different (ischemia or toxins are major factors that precipitate in the injury), but similar pathways may be involved in subsequent injury responses. For this, reason to study AKI models, various methods were defined for each specific situation.

Gentamicin derived from gram-positive bacteria called Micromonospora purpurea present in soil and water having potential in treating aerobic gram-negative bacteria. Accumulation of gentamicin in proximal renal tubules may cause nephrotoxicity which leads to brush border network damage [1]. The nephrotoxicity involves renal free radical production and accumulation, consumption of antioxidant defense mechanisms, glomerular congestion, and acute tubular necrosis [2-5], leading to diminished creatinine clearance and renal dysfunction. The pathological mechanisms also involve elevation of endothelin-1, upregulation of transforming growth factor-beta (TGF- $\beta$ ), significant increase in monocyte/macrophage infiltration into the renal cortex and medulla, augmentation of oxidative stress, and apoptosis and also necrosis [6-9].

Vitamin D is a pleiotropic hormone that affects classical and nonclassical tissues. Its primary sites of action are still considered to be the intestine, bone, and kidneys [10]. A 
number of studies have shown positive therapeutic efficacy of vitamin D and analogs to reduce proteinuria [11-13]. Recently, a large randomized placebo-controlled clinical trial (the VITAL Study, $n=281$ ) confirmed that paricalcitol was able to reduce albuminuria and blood pressure in patients with diabetic nephropathy who were already on renin-angiotensin system inhibitor therapy [14]. Together, these clinical data provide a strong case to argue for the use of vitamin $\mathrm{D}$ analogs as a complementary therapy for treatment of proteinuria. Given the importance of podocytes in the regulation of glomerular filtration, it is speculated that podocytes are important antiproteinuric targets of vitamin D [15] although tubular effect of vitamin $\mathrm{D}$ is still debate.

Inflammation and reactive oxygen substances play an important role on acute kidney injury pathophysiology. Vitamin D has already known antiinflammatory and immunomodulatory effects. In the present study, the aim was to investigate whether vitamin $\mathrm{D}$ might be a useful therapeutic agent for gentamicin-induced AKI model in rats. Up to now vitamin $\mathrm{D}$ related protection mechanisms on AKI remain to be fully proven. Given the complexity of the disease and the pleiotropic nature of the agent activity, the protective effect would be expected and be of a multifactorial nature.

\section{Methods}

2.1. Study Protocol. Thirty nonuremic Wistar albino male rats ( $n=30$; weight $180-220 \mathrm{~g}$ ) were divided into three equal groups. They were housed in polycarbonate cages under $24^{\circ} \mathrm{C}$ room temperature with a 12-hour light/dark cycle and fed a standard laboratory diet. The Animal Ethics Committee of Ege University Hospital approved the study design. The three groups of rats consisted of the following: Control group, $1 \mathrm{~mL}$ saline intramuscular (im) daily; Genta group, gentamicin $100 \mathrm{mg} / \mathrm{kg} /$ day (im); Genta + vitamin D, gentamicin $100 \mathrm{mg} / \mathrm{kg} /$ day $(\mathrm{im})$ in addition to $1 \alpha, 25(\mathrm{OH})_{2} \mathrm{D}_{3}$ $0.4 \mathrm{mcg} / \mathrm{kg} /$ day subcutaneously for 8 days.

Systolic blood pressure was measured in conscious rats by the indirect tail cuff method, using an electrosphygmomanometer and pneumatic pulse transducer (MAY NIBP200-A, Ankara, Turkey), and 24-hour urine was collected in metabolic cages. After 1 hour, ketamine HCL anesthesia ( $60 \mathrm{~mL} / \mathrm{kg}$ body weight) was applied, and immediately, blood samples were collected through direct cardiac puncture in sacrificed rats. Semiquantitative assessment of kidneys was carried out by the same pathologist who was unaware of the groups.

2.2. Functional Parameters. Serum urea and creatinine levels and urine gamma glutamine transferase (GGT) activity were determined using commercial available kits spectrophotometrically (Biolabo Reagents, Maizy France). Urine (Lipocalin-2)-Neutrophil gelatinase-associated lipocalin (NGAL) (Boster Biological Technology Co, Ltd.), and kidney injury molecule-1 (KIM-1) (Adipo Bioscience, Inco, USA) levels were measured with ELISA kits. Urine glutathione (GSH) levels were determined by a commercially available kit (Cayman Chemical Company, Ann Arbor, MI, USA).

2.3. Structural Parameters. Four percent formalin was used for fixation of kidney samples which were embedded into paraffin wax. Paraffin blocks were divided into sections $5 \mu \mathrm{m}$ in thickness, and hematoxylin-eosin and Masson's were trichrome used for staining. All samples were examined by the same pathologist who was unaware of which samples originated from which group. Tubular degeneration, necrosis, tubule interstitial nephritis, and total histological scores were evaluated semiquantitatively from 0 to 3 .

Tubular degeneration (TD): in the cytoplasm of the proximal tubule epithelial cells, stained bodies of various sizes and vacuolization containing acidophilus were considered as TD.

Scoring:

Absence of TD; 0

Mild TD: small and a few focus TD in immediately beneath the capsule $(0 \%-10) ; 1$

Moderate TD: for a few focal focus TD and along the tubular segment (10\%-25); 2

Severe TD: diffuse and significant TD along the tubular segment (\% 25-50); 3

Very severe TD: TD was greater than $50 \% ; 4$

Tubular necrosis (TN): defined as loss of epithelial cells of the nucleus, dark acidophilic cytoplasm, loss of tubular epithelial cells into tubular lumen, and acellular sections of tubules.

Scoring:

Absence of TN; 0

Mild TN: small and a few focus TN in immediately beneath the capsule $(0 \%-10) ; 1$

Moderate TN: for a few focal focus TN and along the tubular segment (10\%-25); 2

Severe TD: diffuse and significant TN along the tubular segment (\% 25-50); 3

Very severe TN: TN was greater than $50 \% ; 4$

Tubulointerstitial inflammation (TIN): defined as infiltration of inflammatory cells in perivascular and interstitial areas.

Scoring:

Absence of TIN; 0

Mild TIN: a few pieces of infiltration concentrated on perivascular area $(0-5 \%) ; 1$

Moderate TIN: usually infiltrations involved in cortical interstitial and many focal areas (5-10\%); 2

Severe TIN: diffuse and significant infiltration areas (15-25\%); 3

Very severe TIN: TIN was greater than $50 \% ; 4$ 


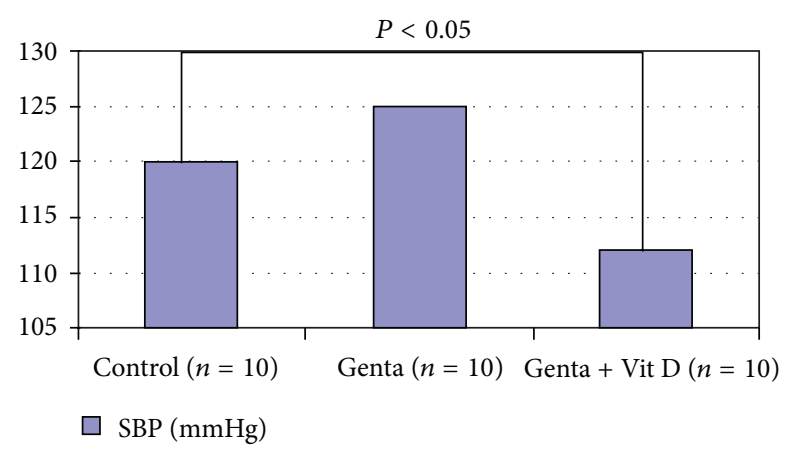

FIGURE 1: Blood pressures.

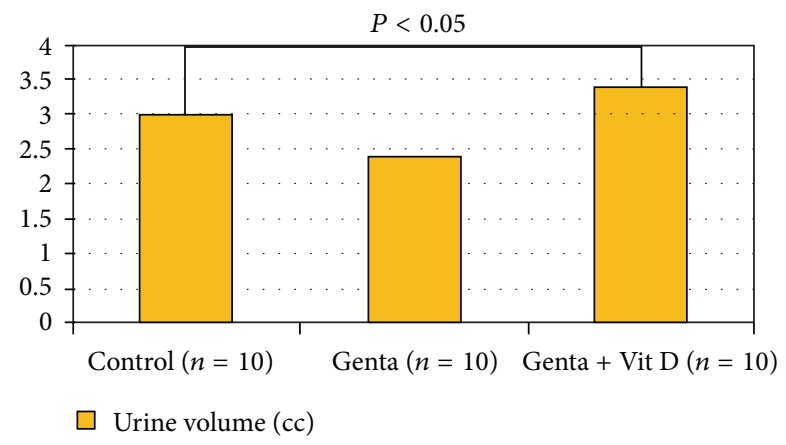

FIgURE 2: Urine Volumes.

Total histologic score (THS): TD/2 + TN + TIN/2, respectively.

\author{
Normal THS: $0-2$ \\ Mild THS: 2-5 \\ Moderate THS: 5-8 \\ Severe TH: $>8$
}

2.4. Statistical Analysis. Study results are presented as mean \pm standard error of the mean (SEM). Nonparametric tests (Kruskal Wallis, Man-Whitney $U$ test) were performed as statistical evaluation, and $P<0.05$ was considered as significant.

\section{Results}

Systolic blood pressure in Control group was $120 \pm 6$ and decreased to $112 \pm 13 \mathrm{mmHg}$ (Figure 1), and urine volume increased in Genta + Vit D group $(3.4 \pm 0.5 \mathrm{cc})$ compared to Control group $(3 \pm 0.5 \mathrm{cc})(P<0.05)$ (Figure 2$)$. In Control group, urea and creatinine levels were $91 \pm 6$ and $0.74 \pm 0.03$ and increased to $137 \pm 6 \mathrm{mg} / \mathrm{dL}$ and $1.1 \pm 0.1 \mathrm{mg} / \mathrm{dL}$ in Genta group, also $149 \pm 5 \mathrm{mg} / \mathrm{dL}$ and $1.6 \pm 0.3 \mathrm{mg} / \mathrm{dL}$ in Genta + Vit D, indicating acute kidney injury (Figure 3 ). Neutrophil gelatinase-associated lipocalin (NGAL) was $49.5 \pm 7$ significantly and increased to $390 \pm 143 \mathrm{ng} / \mathrm{mL}$ in Genta group and decreased to $247 \pm 112 \mathrm{ng} / \mathrm{mL}$ in Genta + Vit D group. Glutathione and gamma glutamine transferase were $0.4 \pm$ 0.13 and $1.3 \pm 0.35$ and increased to $0.6 \pm 0.1 \mathrm{nmol} / \mathrm{mL}$ and $59 \pm 19$ U/L in Genta + Vit D group (Figure 4). Kidney injury
TABLE 1: Clinical and laboratory findings.

\begin{tabular}{lccc}
\hline & Control, $n=10$ & Genta, $n=10$ & $\begin{array}{c}\text { Genta }+ \text { Vit D, } \\
n=10\end{array}$ \\
\hline SBP (mmHg) & $120 \pm 6$ & $125 \pm 10$ & $112 \pm 13^{\mathrm{a}}$ \\
DBP (mmHg) & $68 \pm 4$ & $75 \pm 7$ & $74 \pm 8$ \\
Urine volume (cc) & $3 \pm 0.5$ & $2.4 \pm 0.4$ & $3.4 \pm 0.5^{\mathrm{a}}$ \\
Urea (mg/dL) & $91 \pm 6$ & $137 \pm 6^{\mathrm{a}}$ & $149 \pm 5^{\mathrm{a}}$ \\
Creatinine (mg/dL) & $0.74 \pm 0.03$ & $1.1 \pm 0.1^{\mathrm{a}}$ & $1.6 \pm 0.3^{\mathrm{a}}$ \\
NGAL (ng/mL) & $49.5 \pm 7$ & $390 \pm 143^{\mathrm{a}}$ & $247 \pm 112$ \\
GSH (nmol/mL) & $0.4 \pm 0.13$ & $0.3 \pm 0.04$ & $0.6 \pm 0.1^{\mathrm{a}}$ \\
GGT (U/L) & $1.3 \pm 0.35$ & $38 \pm 37$ & $59 \pm 19^{\mathrm{a}}$ \\
KIM-1 (ng/mL) & $0.64 \pm 0.05$ & $4.7 \pm 0.6^{\mathrm{a}}$ & $6 \pm 0.5^{\mathrm{a}}$ \\
TD & $0.9 \pm 0.1$ & $2 \pm 0^{\mathrm{a}}$ & $2.3 \pm 0.2^{\mathrm{a}}$ \\
TN & 0 & $1.3 \pm 0.2^{\mathrm{a}}$ & $1.2 \pm 0.2^{\mathrm{a}}$ \\
TIN & $0.4 \pm 0.2$ & $1.4 \pm 0.2^{\mathrm{a}}$ & $2 \pm 0.1^{\mathrm{ab}}$ \\
THS & $0.75 \pm 0.15$ & $3.4 \pm 0.4^{\mathrm{a}}$ & $6.3 \pm 0.7^{\mathrm{ab}}$ \\
\hline
\end{tabular}

SBP: systolic blood pressure; DBP: diastolic blood pressure; NGAL: neutrophil gelatinase-associated lipocalin; GSH: glutathione; KIM: kidney injury molecule; TD: tubular degeneration; TN: tubular necrosis; TIN: tubulointerstitial nephritis; THS: total histological score; $P<0.05$, ${ }^{a}$ Group versus Control, ${ }^{\mathrm{b}}$ Group versus Genta.

molecule $1(\mathrm{KIM}-1)$ level was $0.64 \pm 0.05$ in Control group and increased significantly in both Genta and Genta + Vit D groups $(4.7 \pm 0.6$ and $6 \pm 0.5 \mathrm{ng} / \mathrm{mL}$ ) (Table 1 ).

Histological scores of tubular degeneration (TD), tubular necrosis (TN), tubulointerstitial nephritis (TIN), and total histological score (THS) all increased significantly in Genta and Genta + Vit D groups compared to Control group (Figures 5 and 6). TIN and THS scores also significantly were higher in Genta + Vit D group compared to Genta group (Table 1).

\section{Discussion}

Gentamicin is a positively charged chemical that strongly binds to the acidic phosphoinositide components of the brush border membrane which is a negatively charged portion of the proximal tubule, and they mainly act on the cationic drug receptor, megalin, located deeply at the base of the brush border villi. The receptor-drug complex thus formed is rapidly internalized by a pinocytosis process and checked up by lysosomes, where lysosomal phospholipidosis occurs that disrupts a number of renal intracellular processes [16, 17].

Renin angiotensin system (RAS) in the kidney is a mandatory mediator of renal injury. Vitamin D hormone has a negative regulatory effect on RAS by suppressing renin expression $[18,19]$. It is shown that vitamin $\mathrm{D}$ receptor-absent mutant mice develop more severe renal damage (e.g., interstitial fibrosis, increased albuminuria, and glomerulosclerosis) than wild-type counterparts in diabetic state [20] or under postrenal acute kidney injury [21], because of enhanced activation of the RAS in the kidney. In 5/6 nephrectomised rats given paricalcitol treatment attenuated tubulointerstitial and glomerular injury and decreased blood pressure and albuminuria by inhibiting the activation of the locally produced RAS in the remnant kidneys [22]. Doxercalciferol had an effect on modulating fat-induced renal injury by targeting 


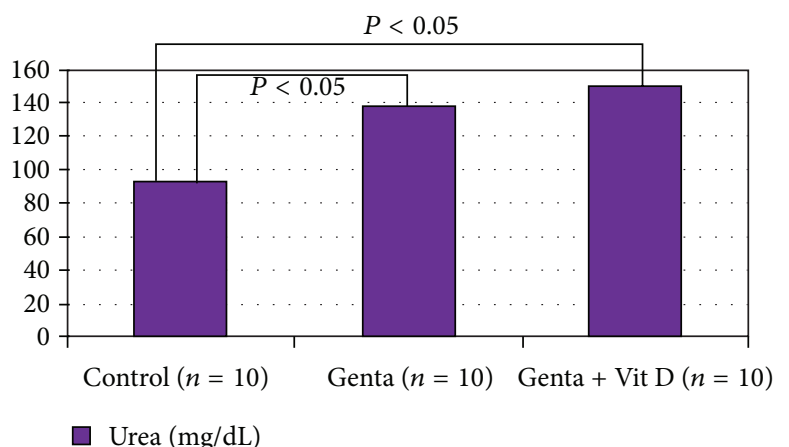

(a)

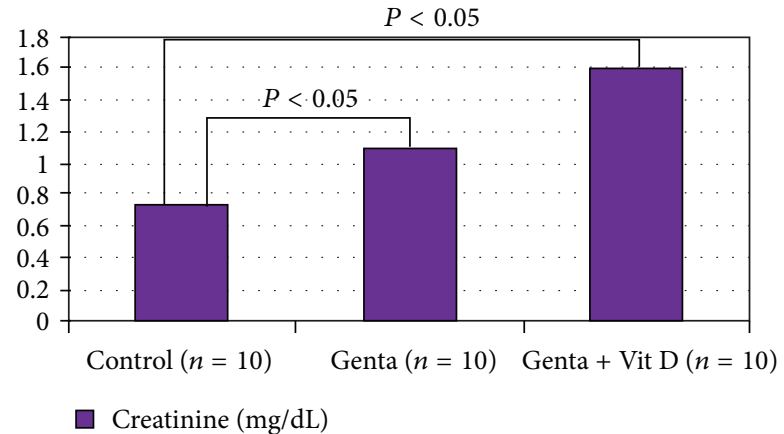

(b)

FIGURE 3: Renal function tests.

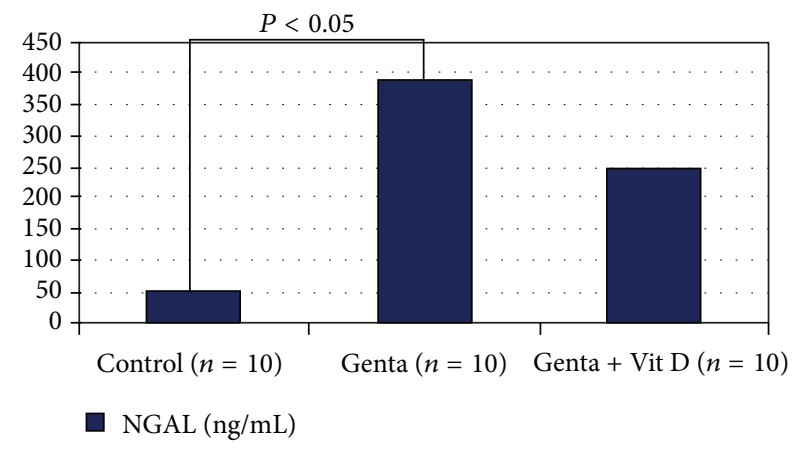

(a)

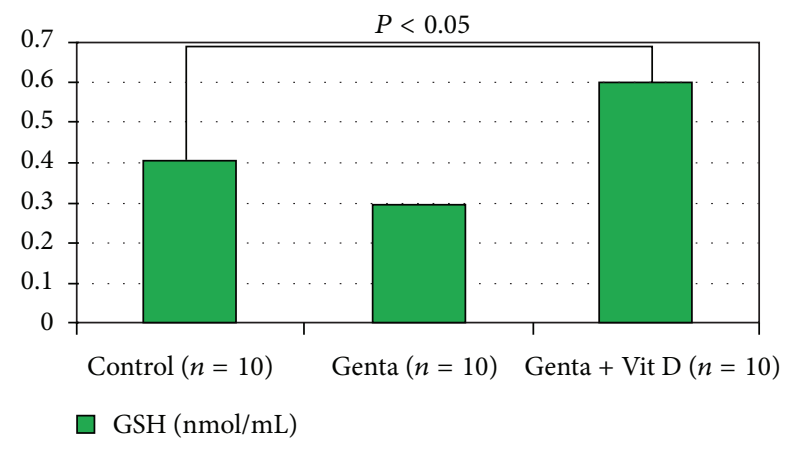

(b)

FIGURE 4: Urine neutrophil gelatinase-associated lipocalin and glutathione levels.

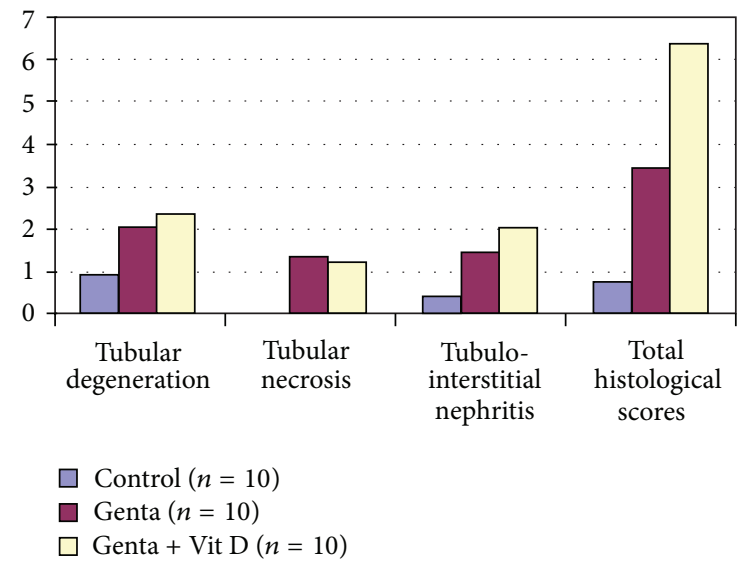

FIgURE 5: Renal histology. Pathological examination performed by semiquantitatively scored from 0 to 3 .

the RAS and lipid metabolism [23]. Other studies proved that combination therapy with one RAS inhibitor (ACE inhibitor or ARB) and one vitamin D analog (paricalcitol or doxercalciferol) leads to additive or synergistic therapeutic effects in blocking renal injury in experimental rat models of type 1 and type 2 diabetes mellitus [24-27]. The renal protection of the combination therapy is the inhibition of the compensatory renin induction usually encountered in the use of both RAS inhibitors and the vitamin D analogues. Renin induction inhibition and accumulation of angiotensin II within the kidney leads to excellent therapeutic results
[28]. The combination strategy in these studies explains why vitamin $\mathrm{D}$ analogs are still effective in reducing albuminuria in CKD patients who are already receiving RAS inhibitors $[29,30]$. In present study, we found that SBP is decreased in Genta + vitamin D group compared to Control group. This indicates that the RAS blocking effect of vitamin D was still strong enough even in the presence of acute kidney injury. Increased urine volume in this group also may be attributed to RAS blocking effect of vitamin D therapy.

Functionally, gentamicin-related nephrotoxicity is characterized by a decrease in glomerular filtration rate and high levels of serum creatinine and blood urea, indicating renal dysfunction [31-37]. In the present study, Genta-induced experimental AKI model is formed and proven by an increase of these renal function tests appropriately. Unfortunately, they were still higher in Genta + vitamin D group than in Control group.

In the literature, there are increasing multifactorial mechanisms suggested as the leading cause of gentamicin nephrotoxicity. Lysosomal apoptosis and phospholipidosis have been suggested to play a pivotal role in gentamicininduced nephrotoxicity [38-40]. In the past, gentamicin was shown to increase reactive oxygen species (ROS) like superoxide anions, hydroxyl radicals and hydrogen peroxides, and reactive nitrogen species generation in the renal cortex that eventually lead to renal structural and functional deterioration [41-44]. Further, it is linked with marked increases in lipid peroxidation levels [45], nitrotyrosine formation [46] and protein oxidation [47]. In our study, we demonstrated 

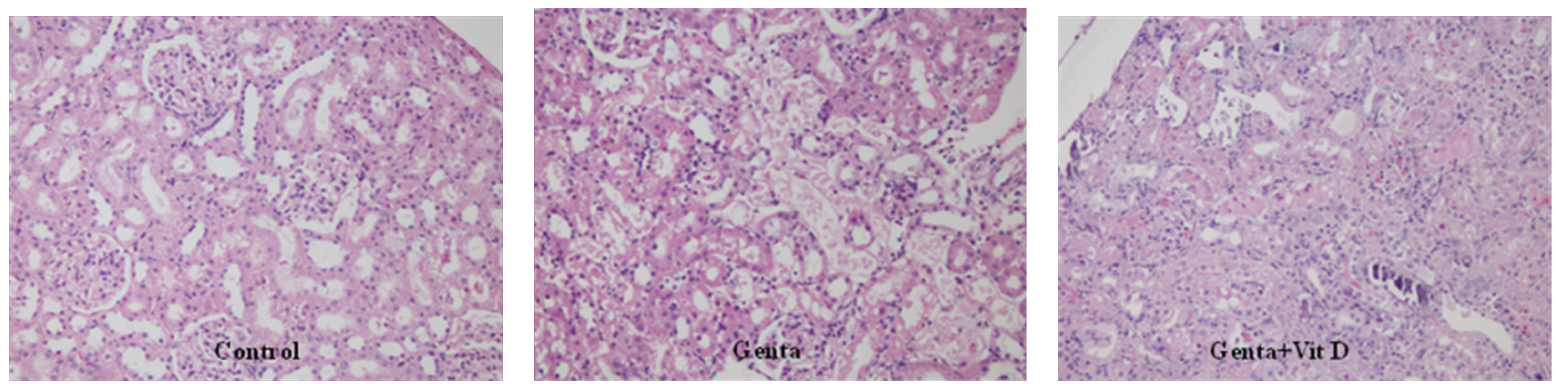

FIgURE 6: Renal pathology.

that in Genta group although it did not reach statistical significance a little GSH decrease occurred. On the other hand, Genta + Vit D group had a statistically significant GSH increase. In the literature, gentamicin has been also shown to cause changes in the composition of lipid membranes executed by free radicals mediated lipid peroxidation [48]. Furthermore, gentamicin-administered rat kidneys are more susceptible to ROS damage because of the induction of deficiency in antioxidant defense enzymes like superoxide dismutase and catalase $[49,50]$. Here in our study, vitamin D might have some beneficial effect on gentamicin-induced AKI by increasing GSH levels and acting as an antioxidant mechanism, and also NGAL levels were not increased unlike to Genta group.

Structurally, gentamicin-related nephrotoxicity is associated with the edema of proximal tubular cells, glomerular hypertrophy, perivascular edema, inflammation, glomerular congestion, cellular desquamation, glomerular atrophy, tubular necrosis, and tubular fibrosis [40, 51-57]. Gentamicin causes macrophage infiltration and higher transforming growth factor- $\beta$ which may lead to progression of TIN [40]. In the present study, TIN scores were significantly higher in Genta group, but surprisingly in Genta + Vit D group, histological scores were even higher than Genta group. Tubular histological parameters all were increased in Genta group indicating experimental AKI model occurred but unfortunately all these parameters were not decreased in Genta + Vit D group.

Acut kidney injury as a result of gentamicin-induced tubular necrosis stimulates inflammatory events by recruiting intercellular adhesion molecule-1 and monocyte chemoattractant protein-1 at the site of injury that enhance the migration of monocytes and macrophages to the site of tissue damage, ultimately leading to renal pathogenesis [58, 59]. In present study we demonstrated that GGT levels in Genta + Vit D group and KIM-1 levels in both Genta and Genta + Vit $D$ groups were increased indicating that renal tubular damage occurred in Genta groups and also even using Vit D did not prevent progression of injury.

\section{Conclusion}

In the past, vitamin $\mathrm{D}$ was shown as an effective drug on podocytes preventing proteinuria, regulate bone remodeling, regulate cell cycles, and the renin-angiotensin system [60]. The present study indicates that the progression of gentamicin-induced AKI was not stopped by vitamin D treatment shown by histological findings although it probably has some beneficial effects on the RAS system via blood pressure lowering and increase of urine volume and a promising effect on antioxidant system. As a result given the various overlapping pathways involved in AKI pathogenesis, intended therapies may need to use vitamin $\mathrm{D}$ in addition to other therapeutical approaches to target diverse pathways in order to achieve success.

\section{Disclosure}

The results presented in this paper have not been published previously in whole or part, presented in abstract form. All the authors have contributed substantially to the research, preparation and production of the paper and approve of its submission to the Journal.

\section{References}

[1] P. H. Whiting and P. A. J. Brown, "The relationship between enzymuria and kidney enzyme activities in experimental gentamicin nephrotoxicity," Renal Failure, vol. 18, no. 6, pp. 899909, 1996.

[2] M. P. Mingeot-Leclercq and P. M. Tulkens, "Aminoglycosides: nephrotoxicity," Antimicrobial Agents and Chemotherapy, vol. 43, no. 5, pp. 1003-1012, 1999.

[3] A. A. Elfarra, R. J. Duescher, P. J. Sausen, T. M. O'Hara, and A. J. Cooley, "Methimazole protection of rats against gentamicininduced nephrotoxicity," Canadian Journal of Physiology and Pharmacology, vol. 72, no. 10, pp. 1238-1244, 1994.

[4] T. J. Geleilete, G. C. Melo, R. S. Costa, R. A. Volpini, T. J. Soares, and T. M. Coimbra, "Role of myofibroblasts, macrophages, transforming growth factor- $\beta$ endothelin, angiotensin-II, and fibronectin in the progression of tubulointerstitial nephritis induced by gentamicin," Journal of Nephrology, vol. 15, no. 6, pp. 633-642, 2002.

[5] I. T. Abdel-Raheem, A. A. Abdel-Ghany, and G. A. Mohamed, "Protective effect of quercetin against gentamicin-induced nephrotoxicity in rats," Biological and Pharmaceutical Bulletin, vol. 32, no. 1, pp. 61-67, 2009.

[6] B. H. Ali, "Gentamicin nephrotoxicity in humans and animals: some recent research," General Pharmacology, vol. 26, no. 7, pp. 1477-1487, 1995.

[7] T. Finkel, "Oxygen radicals and signaling," Current Opinion in Cell Biology, vol. 10, no. 2, pp. 248-253, 1998. 
[8] M. El Mouedden, G. Laurent, M. Mingeot-Leclercq, H. S. Taper, J. Cumps, and P. M. Tulkens, "Apoptosis in renal proximal tubules of rats treated with low doses of aminoglycosides," Antimicrobial Agents and Chemotherapy, vol. 44, no. 3, pp. 665$675,2000$.

[9] G. Bledsoe, S. Crickman, J. Mao et al., "Kallikrein/kinin protects against gentamicin-induced nephrotoxicity by inhibition of inflammation and apoptosis," Nephrology Dialysis Transplantation, vol. 21, no. 3, pp. 624-633, 2006.

[10] C. J. Rosen, J. S. Adams, D. D. Bikle et al., "The nonskeletal effects of vitamin D: an endocrine society scientific statement," vol. 33, no. 3, pp. 456-492, 2012.

[11] R. Agarwal, M. Acharya, J. Tian et al., "Antiproteinuric effect of oral paricalcitol in chronic kidney disease," Kidney International, vol. 68, no. 6, pp. 2823-2828, 2005.

[12] P. Alborzi, N. A. Patel, C. Peterson et al., "Paricalcitol reduces albuminuria and inflammation in chronic kidney disease a randomized double-blind pilot trial," Hypertension, vol. 52, no. 2, pp. 249-255, 2008.

[13] S. Fishbane, H. Chittineni, M. Packman, P. Dutka, N. Ali, and N. Durie, "Oral paricalcitol in the treatment of patients with CKD and proteinuria: a randomized trial," The American Journal of Kidney Diseases, vol. 54, no. 4, pp. 647-652, 2009.

[14] D. de Zeeuw, R. Agarwal, M. Amdahl et al., "Selective vitamin $\mathrm{D}$ receptor activation with paricalcitol for reduction of albuminuria in patients with type 2 diabetes (VITAL study): a randomised controlled trial," The Lancet, vol. 376, no. 9752, pp. 1543-1551, 2010.

[15] Y. C. Li, "Podocytes as target of vitamin D," Current Diabetes Reviews, vol. 7, no. 1, pp. 35-40, 2011.

[16] G. Laurent, M. B. Carlier, B. Rollman, F. van Hoof, and P. Tulkens, "Mechanism of aminoglycoside-induced lysosomal phospholipidosis: in vitro and in vivo studies with gentamicin and amikacin," Biochemical Pharmacology, vol. 31, no. 23, pp. 3861-3870, 1982.

[17] J. S. Sandhu, A. Sehgal, O. Gupta, and A. Singh, "Aminoglycoside nephrotoxicity revisited," Journal of Indian Academy of Clinical Medicine, vol. 8, no. 4, pp. 331-333, 2007.

[18] Y. C. Li, J. Kong, M. Wei, Z. Chen, S. Q. Liu, and L. Cao, "1,25dihydroxyvitamin D3 is a negative endocrine regulator of the renin-angiotensin system," Journal of Clinical Investigation, vol. 110, no. 2, pp. 229-238, 2002.

[19] W. Yuan, W. Pan, J. Kong et al., "1,25-dihydroxyvitamin D3 suppresses renin gene transcription by blocking the activity of the cyclic AMP response element in the renin gene promoter," The Journal of Biological Chemistry, vol. 282, no. 41, pp. 2982129830, 2007.

[20] Z. Zhang, L. Sun, Y. Wang et al., "Renoprotective role of the vitamin D receptor in diabetic nephropathy," Kidney International, vol. 73, no. 2, pp. 163-171, 2008.

[21] Y. Zhang, J. Kong, D. K. Deb, A. Chang, and Y. C. Li, "Vitamin D receptor attenuates renal fibrosis by suppressing the renin-angiotensin system," Journal of the American Society of Nephrology, vol. 21, no. 6, pp. 966-973, 2010.

[22] M. Freundlich, Y. Quiroz, Z. Zhang et al., "Suppression of reninangiotensin gene expression in the kidney by paricalcitol," Kidney International, vol. 74, no. 11, pp. 1394-1402, 2008.

[23] X. X. Wang, T. Jiang, Y. Shen et al., "Vitamin D receptor agonist doxercalciferol modulates dietary fat-induced renal disease and renal lipid metabolism," The American Journal of Physiology, vol. 300, no. 3, pp. F801-F810, 2011.
[24] Z. Zhang, Y. Zhang, G. Ning, D. K. Deb, J. Kong, and C. L. Yan, "Combination therapy with AT1 blocker and vitamin D analog markedly ameliorates diabetic nephropathy: blockade of compensatory renin increase," Proceedings of the National Academy of Sciences of the United States of America, vol. 105, no. 41, pp. 15896-15901, 2008.

[25] D. K. Deb, T. Sun, K. E. Wong et al., "Combined vitamin D analog and AT1 receptor antagonist synergistically block the development of kidney disease in a model of type 2 diabetes," Kidney International, vol. 77, no. 11, pp. 1000-1009, 2010.

[26] Y. Zhang, D. K. Deb, J. Kong et al., "Long-term therapeutic effect of vitamin $\mathrm{D}$ analog doxercalciferol on diabetic nephropathy: strong synergism with AT1 receptor antagonist," The American Journal of Physiology, vol. 297, no. 3, pp. F791-F801, 2009.

[27] I. Ohara, M. Tanimoto, T. Gohda et al., "Effect of combination therapy with angiotensin receptor blocker and 1,25dihydroxyvitamin D3 in type 2 diabetic nephropathy in KKAy/Ta mice," Nephron-Experimental Nephrology, vol. 117, no. 4, pp. e124-e132, 2011.

[28] Y. C. Li, "Renoprotective effects of vitamin D analogs," Kidney International, vol. 78, no. 2, pp. 134-139, 2010.

[29] P. Alborzi, N. A. Patel, C. Peterson et al., "Paricalcitol reduces albuminuria and inflammation in chronic kidney disease: a randomized double-blind pilot trial," Hypertension, vol. 52, no. 2, pp. 249-255, 2008.

[30] D. de Zeeuw, R. Agarwal, M. Amdahl et al., "Selective vitamin D receptor activation with paricalcitol for reduction of albuminuria in patients with type 2 diabetes (VITAL study): a randomised controlled trial," The Lancet, vol. 376, no. 9752, pp. 1543-1551, 2010.

[31] F. Romero, M. Pérez, M. Chávez, G. Parra, and P. Durante, "Effect of uric acid on gentamicin-induced nephrotoxicity in rats-role of matrix metalloproteinases 2 and 9," Basic and Clinical Pharmacology and Toxicology, vol. 105, no. 6, pp. 416424, 2009.

[32] O. A. Al-Shabanah, A. M. Aleisa, A. A. Al-Yahya et al., "Increased urinary losses of carnitine and decreased intramitochondrial coenzyme A in gentamicin-induced acute renal failure in rats," Nephrology Dialysis Transplantation, vol. 25, no. 1, pp. 69-76, 2010.

[33] E. S. El Daly, "Effect of methimazole and fish oil treatment on gentamicin nephrotoxicity in rats," Journal de Pharmacie de Belgique, vol. 52, no. 4, pp. 149-156, 1997.

[34] J. Pedraza-Chaverrí, P. D. Maldonado, D. Barrera, A. Cerón, O. N. Medina-Campos, and R. Hernández-Pando, "Protective effect of diallyl sulfide on oxidative stress and nephrotoxicity induced by gentamicin in rats," Molecular and Cellular Biochemistry, vol. 254, no. 1-2, pp. 125-130, 2003.

[35] P. Balakumar, A. Rohilla, and A. Thangathirupathi, "Gentamicin-induced nephrotoxicity: do we have a promising therapeutic approach to blunt it?" Pharmacological Research, vol. 62, no. 3, pp. 179-186, 2010.

[36] C. Silan, O. Uzun, N. U. Çomunoğlu, S. Gokçen, S. Bedirhan, and M. Cengiz, "Gentamicin-induced nephrotoxicity in rats ameliorated and healing effects of resveratrol," Biological and Pharmaceutical Bulletin, vol. 30, no. 1, pp. 79-83, 2007.

[37] K. M. Soliman, M. Abdul-Hamid, and A. I. Othman, "Effect of carnosine on gentamicin-induced nephrotoxicity," Medical Science Monitor, vol. 13, no. 3, pp. BR73-BR83, 2007.

[38] G. Laurent, P. Maldague, M. B. Carlier, and P. Tulkens, "Increased renal DNA synthesis in vivo after administration 
of low doses of gentamicin to rats," Antimicrobial Agents and Chemotherapy, vol. 24, no. 4, pp. 586-593, 1983.

[39] M. El Mouedden, G. Laurent, M. Mingeot-Leclercq, H. S. Taper, J. Cumps, and P. M. Tulkens, "Apoptosis in renal proximal tubules of rats treated with low doses of aminoglycosides," Antimicrobial Agents and Chemotherapy, vol. 44, no. 3, pp. 665$675,2000$.

[40] G. Bledsoe, S. Crickman, J. Mao et al., "Kallikrein/kinin protects against gentamicin-induced nephrotoxicity by inhibition of inflammation and apoptosis," Nephrology Dialysis Transplantation, vol. 21, no. 3, pp. 624-633, 2006.

[41] P. D. Walker, Y. Barri, and S. V. Shah, "Oxidant mechanisms in gentamicin nephrotoxicity," Renal Failure, vol. 21, no. 3-4, pp. 433-442, 1999.

[42] P. D. Maldonado, D. Barrera, I. Rivero et al., "Antioxidant Sallylcysteine prevents gentamicin-induced oxidative stress and renal damage," Free Radical Biology and Medicine, vol. 35, no. 3, pp. 317-324, 2003.

[43] Z. Wang, L. Liu, Q. Mei, L. Liu, Y. Ran, and R. Zhang, "Increased expression of heat shock protein 72 protects renal proximal tubular cells from gentamicin-induced injury," Journal of Korean Medical Science, vol. 21, no. 5, pp. 904-910, 2006.

[44] P. Balakumar, V. A. Chakkarwar, V. Kumar, A. Jain, J. Reddy, and M. Singh, "Experimental models for nephropathy," Journal of the Renin-Angiotensin-Aldosterone System, vol. 9, no. 4, pp. 189-195, 2008.

[45] B. H. Ali, "The effect of treatment with the medicinal plant Rhazya stricta decne on gentamicin nephrotoxicity in rats," Phytomedicine, vol. 9, no. 5, pp. 385-389, 2002.

[46] S. Cuzzocrea, E. Mazzon, L. Dugo et al., "A role for superoxide in gentamicin-mediated nephropathy in rats," European Journal of Pharmacology, vol. 450, no. 1, pp. 67-76, 2002.

[47] G. Sener, A. O. Sehirli, H. Z. Altunbas et al., "Melatonin protects against gentamicin-induced nephrotoxicity in rats," Journal of Pineal Research, vol. 32, no. 4, pp. 231-236, 2002.

[48] H. Parlakpinar, S. Tasdemir, A. Polat et al., "Protective role of caffeic acid phenethyl ester (cape) on gentamicin-induced acute renal toxicity in rats," Toxicology, vol. 207, no. 2, pp. 169-177, 2005.

[49] J. Pedraza-Chaverrí, P. D. Maldonado, O. N. Medina-Campos et al., "Garlic ameliorates gentamicin nephrotoxicity: relation to antioxidant enzymes," Free Radical Biology and Medicine, vol. 29, no. 7, pp. 602-611, 2000.

[50] P. D. Maldonado, D. Barrera, O. N. Medina-Campos, R. Hernández-Pando, M. E. Ibarra-Rubio, and J. PedrazaChaverrí, "Aged garlic extract attenuates gentamicin induced renal damage and oxidative stress in rats," Life Sciences, vol. 73, no. 20, pp. 2543-2556, 2003.

[51] A. B. Abdel-Naim, M. H. Abdel-Wahab, and F. F. Attia, "Protective effects of vitamin $\mathrm{E}$ and probucol against gentamicininduced nephrotoxicity in rats," Pharmacological Research, vol. 40, no. 2, pp. 183-187, 1999.

[52] A. Shirwaikar, S. Malini, and S. C. Kumari, "Protective effect of Pongamia pinnata flowers against cisplatin and gentamicin induced nephrotoxicity in rats," Indian Journal of Experimental Biology, vol. 41, no. 1, pp. 58-62, 2003.

[53] A. Ateş̧̧ahin, I. Karahan, S. Yilmaz, A. O. Çeribaşi, and I. Princci, "The effect of manganese chloride on gentamicininduced nephrotoxicity in rats," Pharmacological Research, vol. 48, no. 6, pp. 637-642, 2003.
[54] A. Polat, H. Parlakpinar, S. Tasdemir et al., "Protective role of aminoguanidine on gentamicin-induced acute renal failure in rats," Acta Histochemica, vol. 108, no. 5, pp. 365-371, 2006.

[55] E. Ozbek, M. Cekmen, Y. O. Ilbey, A. Simsek, E. C. Polat, and A. Somay, "Atorvastatin prevents gentamicin-induced renal damage in rats through the inhibition of p38-MAPK and NF- $\kappa \mathrm{B}$ pathways," Renal Failure, vol. 31, no. 5, pp. 382-392, 2009.

[56] I. T. Abdel-Raheem, G. A. El-Sherbiny, and A. Taye, "Green tea ameliorates renal oxidative damage induced by gentamicin in rats," Pakistan Journal of Pharmaceutical Sciences, vol. 23, no. 1, pp. 21-28, 2010.

[57] B. V. S. Lakshmi and M. Sudhakar, "Protective effect of Zingiber officinale on gentamicin-induced nephrotoxicity in rats," International Journal of Pharmacology, vol. 6, no. 1, pp. 58-62, 2010.

[58] S. M. Wahl, D. A. Hunt, and L. M. Wakefield, "Transforming growth factor type $\beta$ induces monocyte chemotaxis and growth factor production," Proceedings of the National Academy of Sciences of the United States of America, vol. 84, no. 16, pp. 57885792, 1987.

[59] W. W. Tang, L. Feng, J. C. Mathison, and C. B. Wilson, "Cytokine expression, upregulation of intercellular adhesion molecule1 , and leukocyte infiltration in experimental tubulointerstitial nephritis," Laboratory Investigation, vol. 70, no. 5, pp. 631-638, 1994.

[60] I. Yildirim, K. Magden, and E. Hur, "Infection inflammation and vitamin D," Clinical Microbiology, vol. 2, article 116, 2013. 


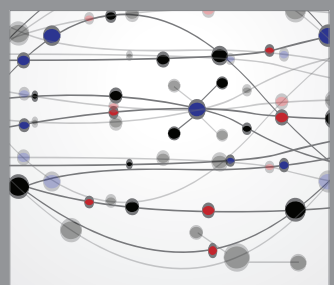

The Scientific World Journal
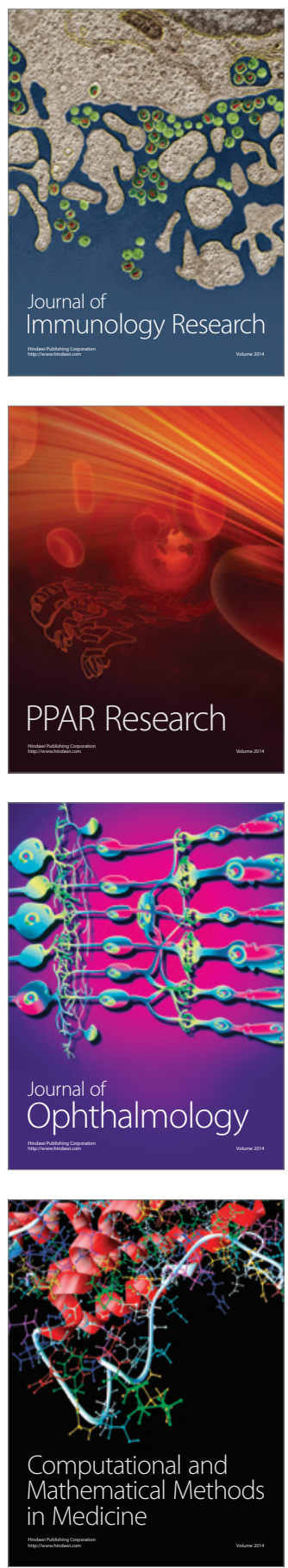

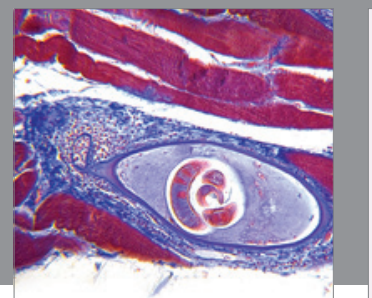

Gastroenterology

Research and Practice
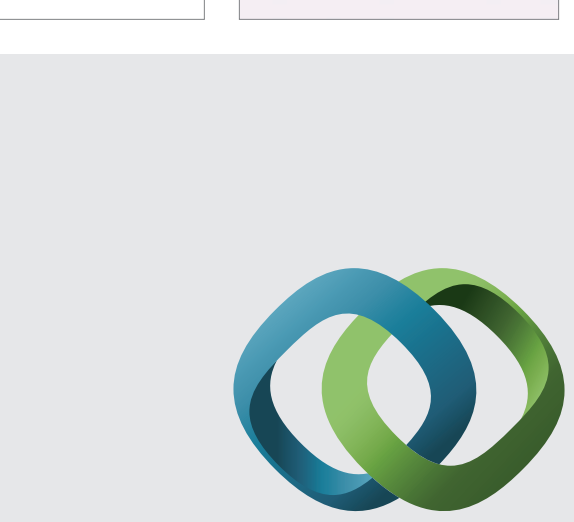

\section{Hindawi}

Submit your manuscripts at

http://www.hindawi.com
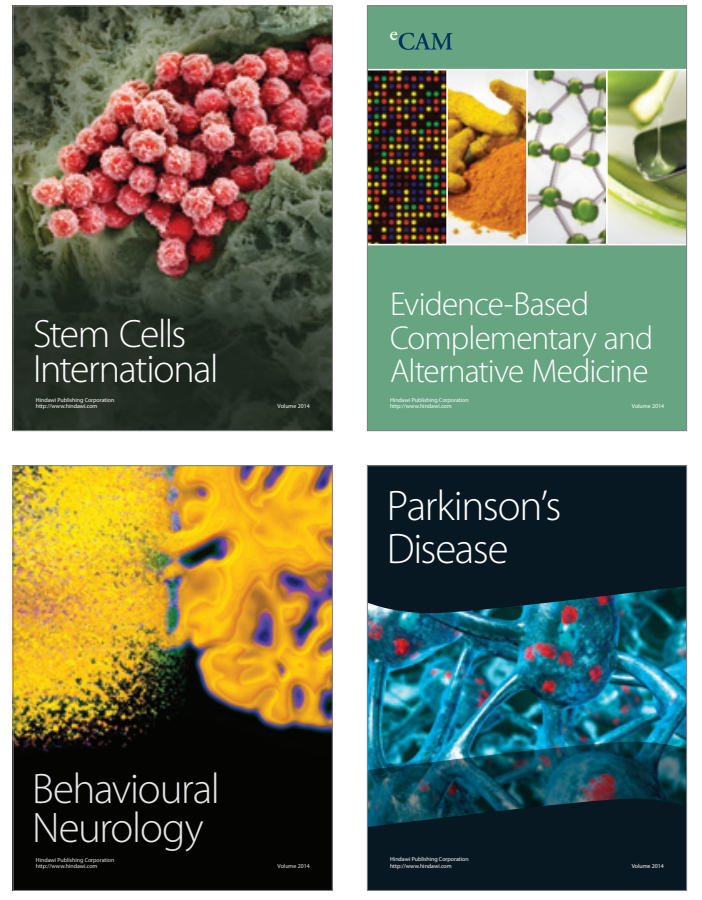
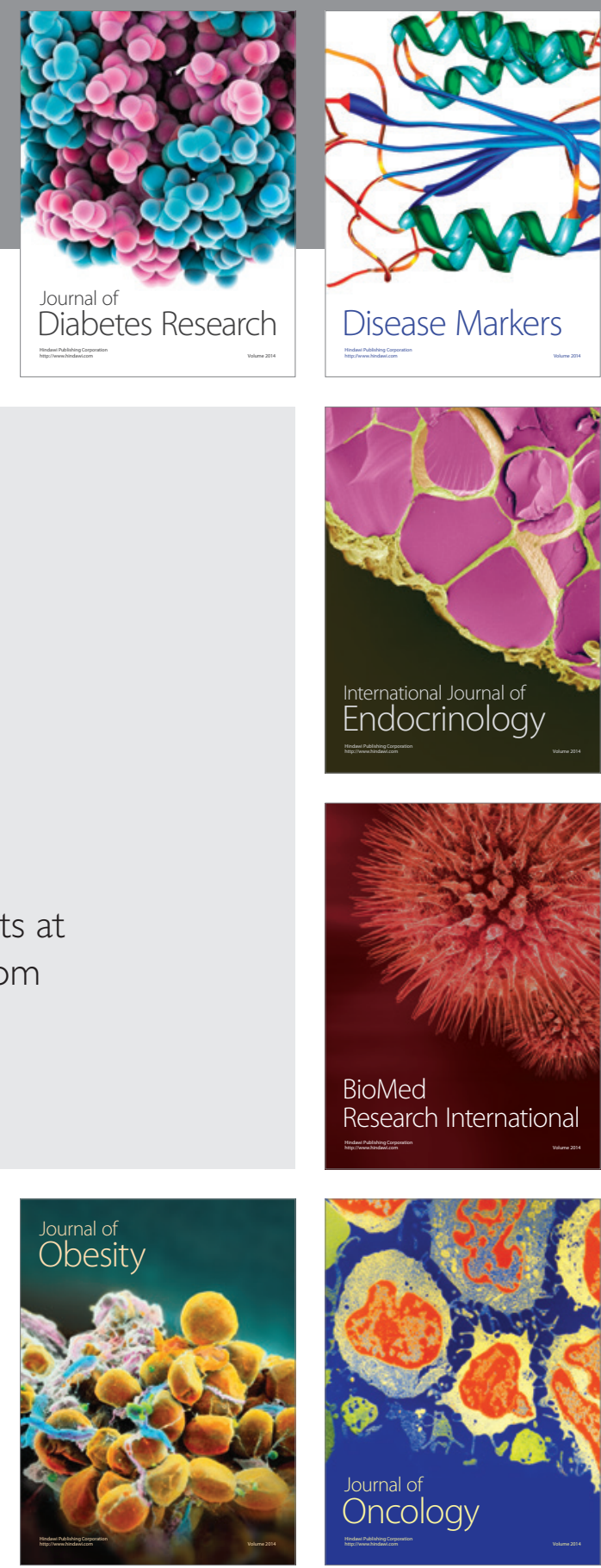

Disease Markers
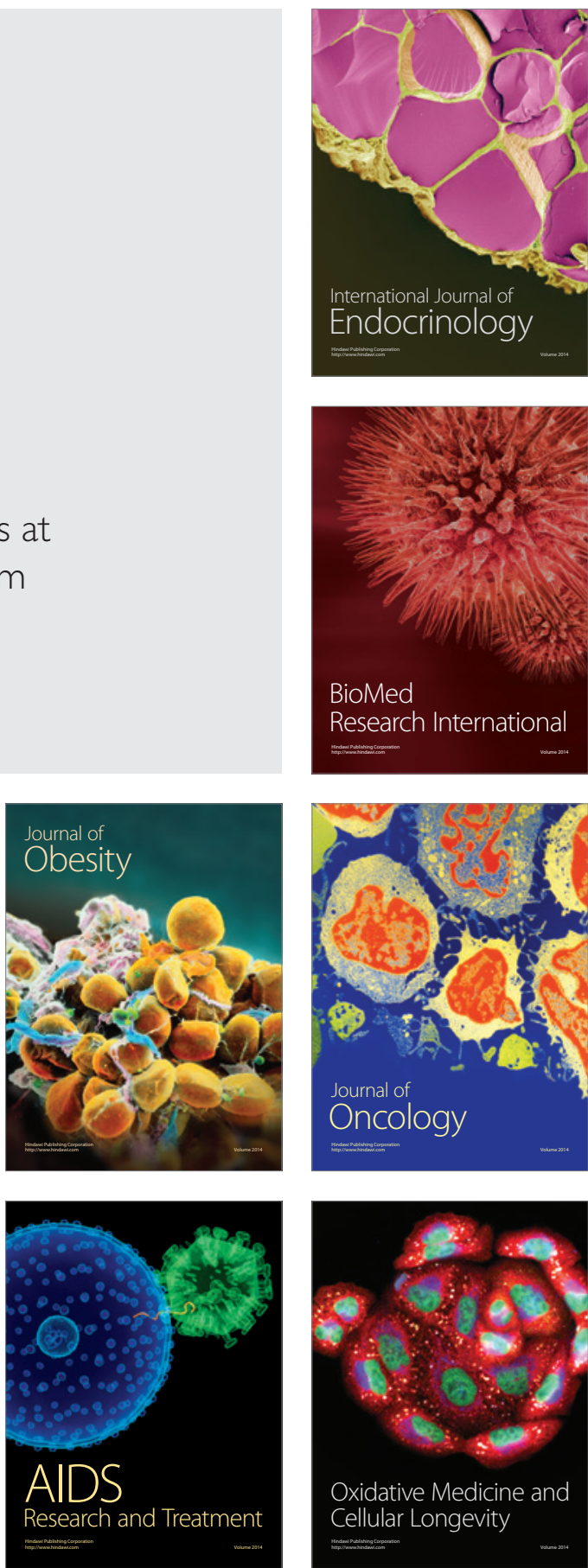